\title{
Compression des cavités cardiaques par une hernie diaphragmatique
}

\section{Compression of the Heart by a Diaphragmatic Hernia}

\section{R. Girerd · T. Bastard}

Reçu le 6 juillet 2015; accepté le 28 juillet 2015

(C) SFMU et Lavoisier SAS 2015

Une patiente de 82 ans, aux antécédents d'hypertension artérielle et de reflux gastro-œsophagien, est adressée par son médecin traitant pour vomissements alimentaires depuis 24 heures. À la prise en charge, la patiente est tachycarde à $150 / \mathrm{min}$, la pression artérielle à $120 / 80 \mathrm{mmHg}$, la saturation à $96 \%$ en air ambiant, une polypnée à $30 / \mathrm{min}$. L'examen clinique retrouve une diminution du murmure vésiculaire à gauche associée à des signes d'insuffisance cardiaque globale. Très rapidement, l'état clinique de la patiente se dégrade avec apparition d'une oxygénodépendance marquée (saturation à $92 \%$ sous $9 \mathrm{~L} / \mathrm{min}$ ) avec une agitation psychomotrice. Une tomodensitométrie thoracique sans injection est réalisée en urgence, qui retrouve une volumineuse hernie diaphragmatique responsable d'une compression des cavités cardiaques. L'évolution va être fatale sur la table du scanner malgré la pose d'une sonde nasogastrique et d'une réanimation spécialisée. La compression des cavités cardiaques par hernie diaphragmatique est une cause rare de dyspnée. Ce diagnostic peut être évoqué sur un cliché thoracique (non réalisé ici) et confirmé par une tomodensitométrie thoracique. Son traitement consiste initialement en une vidange du liquide gastrique puis en une prise en charge chirurgicale.

R. Girerd $(\bowtie) \cdot$ T. Bastard

Service des urgences, centre hospitalier Gabriel Martin,

38 rue Labourdonnais, F-97460 Saint Paul, Réunion

e-mail : remigirerd@gmail.com

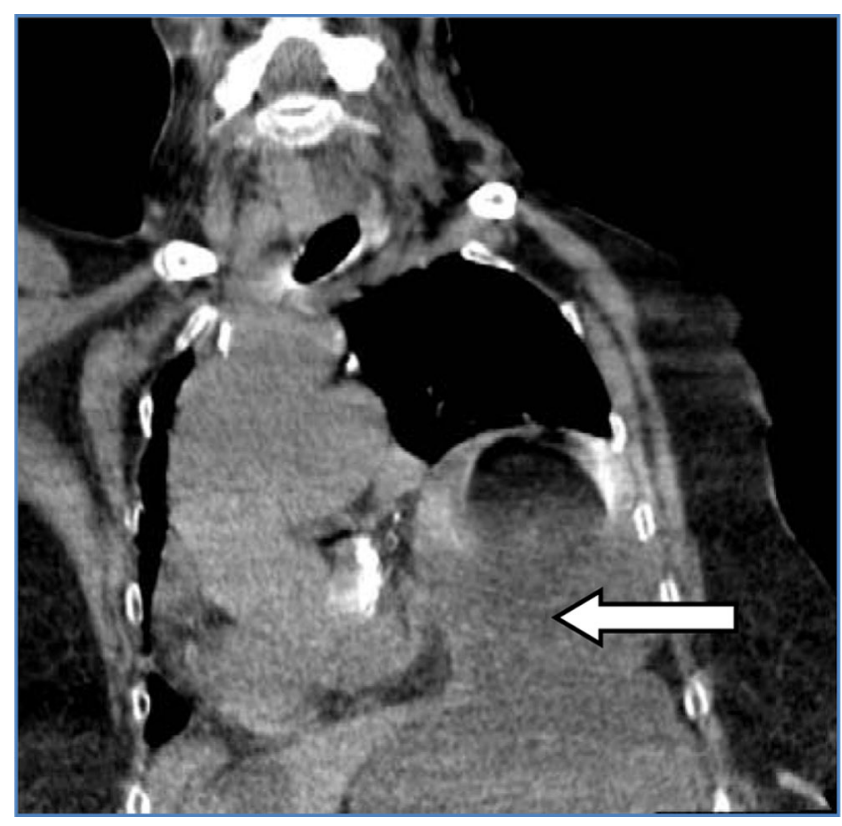

Fig. 1 Tomodensitométrie thoracique sans injection : coupe frontale montrant une hernie diaphragmatique (flèche) responsable d'une compression des cavités cardiaques vers la droite 